\title{
Optimum synthetic-aperture imaging of extended astronomical objects
}

\author{
Casper van der Avoort, Silvania F. Pereira, and Joseph J. M. Braat \\ Optics Research Group, Delft University of Technology, Lorentzweg 1, NL-2628 CJ, Delft, The Netherlands \\ Jan-Willem den Herder \\ SRON, Netherlands Institute for Space Research, Sorbonnelaan 2, NL-3584 CA, Utrecht, The Netherlands
}

Received April 12, 2006; revised August 22, 2006; accepted October 6, 2006; posted October 31, 2006 (Doc. ID 69867); published March 14, 2007

\begin{abstract}
In optical aperture-synthesis imaging of stellar objects, different beam combination strategies are used and proposed. Coaxial Michelson interferometers are very common and a homothetic multiaxial interferometer is recently realized in the Large Binocular Telescope. Laboratory experiments have demonstrated the working principles of two new approaches: densified pupil imaging and wide field-of-view (FOV) coaxial imaging using a staircase-shaped mirror. We develop a common mathematical formulation for direct comparison of the resolution and noise sensitivity of these four telescope configurations for combining beams from multiple apertures for interferometric synthetic aperture, wide-FOV imaging. Singular value decomposition techniques are used to compare the techniques and observe their distinct signal-to-noise ratio behaviors. We conclude that for a certain chosen stellar object, clear differences in performance of the imagers are identifiable. () 2007 Optical Society of America
\end{abstract}

OCIS codes: $110.1650,110.4980,110.5100,110.6770,350.1260,350.6090$.

\section{INTRODUCTION}

For a long time, aperture synthesis imaging in the optical domain was impractical because of the extremely tight tolerances in bringing the light from the various subapertures to a coherent focus. But important technological advances have been made in the past decade, such as adaptive optics and active mechanical control, and optical long baseline stellar interferometry is an astronomical observation technique that has come of age. ${ }^{1,2}$ Only a few of the interferometric telescope arrays currently in operation are designed to perform imaging tasks, as are the cases of the Large Binocular Telescope (LBT) and the Cambridge Optical Aperture Synthesis Telescope (COAST) array., The arrays forming the synthetic aperture are imagers as well as arrays measuring stellar properties, and they perform intensity measurements that, due to the coherence function of the stellar object projected onto the array aperture, will change with the configuration of the array, i.e., the separation and locations of the individual telescopes. For imaging interferometers that combine the beams from telescopes coaxially, measuring the complex degree of coherence allows aperture synthesis imaging as with radio telescope arrays. For multiple aperture imagers with focal plane combination, such as in development for Earth observation but also in astronomy-as studied for the Very Large Telescope Interferometer (VLTI) and realized in the LBT-the postdetection image reconstruction is rather a joint deconvolution operation than a postprocessing of recorded complex degrees of coherence.

In this paper, we describe an approach to compare the imaging performance of both of these types of imagers for a wide-field stellar observation. Moreover, two alternative methods are included in the comparison: densified pupil imaging ${ }^{5}$ and coaxial staircase imaging. ${ }^{6}$ The treatment of partial coherence as well as deconvolution are both covered, unifying the analysis for all types of imaging interferometers.

The examples given in this paper are all onedimensional and treat only two-element interferometers. All simulated observations are polychromatic, with wavelengths $\lambda$ ranging from 6 to $10 \mu \mathrm{m}$. Light of a finite bandwidth results in a certain coherence length; two beams of light with a path length difference larger than this coherence length will not show interference. It should be noticed that partial coherence refers to the fact that light originating from a range of directions is considered, in this case provided by an extended stellar source. Even monochromatically, the observation of such a source is partially coherent with a degree of coherence that is a function of, among other things, the separation of the telescopes. The work presented in this paper was carried out in the framework of the design of an imaging beam combiner for the European Space Agency's (ESA's) DARWIN mission. ${ }^{7}$

\section{DISCRETIZATION OF THE INTERFEROMETRIC IMAGING PROCESS}

Optical interferometric imaging of stellar objects consists in reproducing a luminous intensity function as it appears on the sky from recorded intensity information. The angular spatial frequencies in this luminous intensity function are much higher than the maximum spatial frequency that any of the subapertures in the array can 

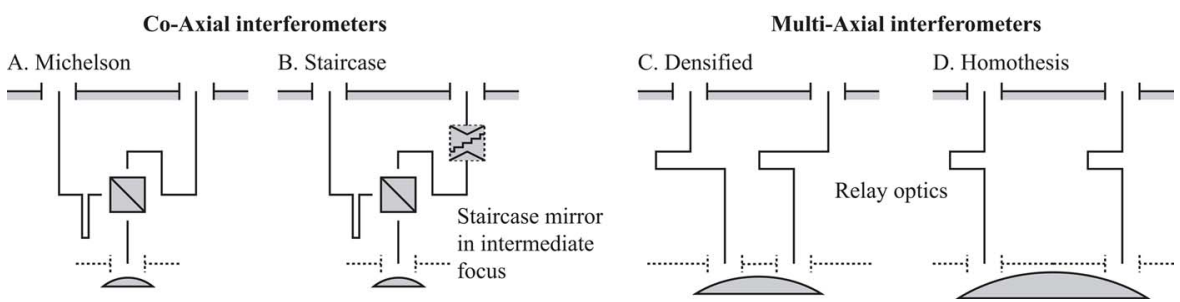

Fig. 1. (Color online) Sketches of four stellar interferometers as described in the literature. Note that Michelson's experiments on stellar interferometry were performed with a configuration similar to sketch $\mathrm{C}$. The label MI as used here and in the literature refers to the coaxial MI-Morley interferometer. Configuration D is commonly addressed as a Fizeau interferometer, but it should be noted that Fizeau experimented with a masked aperture; no beam relay optics were needed.

transfer. Aperture synthesis as known from radioastronomy relies on numerical correlation of recorded electrical fields at each telescope. Optically, these fields cannot be recorded; only the intensity is available. Optical aperture synthesis hence requires correlation of the fields in situ resulting in intensity patterns that can be recorded.

The analytical description of such an intensity pattern is straightforward when monochromatic plane waves originating from a point source arrive at the array. Based on array configuration, an intensity pattern with either spatial fringes or a varying intensity as a function of optical path-length difference or delay can be predicted. Even when many wavelengths are taken into account, the description of the intensity output is insufficient to describe the correct intensity modulation of the output. Michelson (MI) demonstrated that when a star is the source of light, the modulation of the fringed intensity pattern depends on the separation of the subapertures of the array. ${ }^{8,9}$ For different stars, a different relation between separation and modulation is found. The ZernikeVan Cittert theorem ${ }^{10}$ shows that the complex modulation (amplitude and phase), which is referred to as complex degree of coherence, can be predicted by Fourier transforming the luminous intensity distribution function of the source. With the theory of partial coherence, see Born and Wolf, ${ }^{10}$ the propagation of the complex degree of coherence through an optical system can be performed, in order to predict the intensity output.

For polychromatic wide field observations (where wide field is defined as an observation wider than a single incoherent diffraction envelope) the approach based on propagation of partial coherence is possible but elaborate. When the optical recombination scheme of a telescope array incorporates discontinuous reflecting surfaces or transportation (explanation will follow) of partial wavefronts, an analytical description of the intensity output based on diffractive calculations and the propagation of partial coherence is very inefficient. Besides that, physical insight into the imaging process is lost. This insight clarifies and facilitates the inversion of recorded intensity patterns to reconstruct the luminous intensity function on the sky-the main goal of the description of the intensity output.

The method presented in this paper describes analytically defined point-source intensity outputs of four interferometers that are sketched in Fig. 1. Since a stellar source is a collection of independent point sources, the intensity outputs can be summed, resulting in intensity profiles that have a modulation amplitude and phase in accordance with the theory of partial coherence. The summation is performed by a matrix-vector multiplication. We will try to invert this equation at best, with the result that measured intensity profiles can be used to reconstruct a stellar luminous intensity function.

\section{A. Response Functions}

Before constructing a transfer matrix for an interferometric array that can be used to calculate the detector response of an extended stellar object, the intensity pointsource response is derived for that array. A point source at an angle of incidence $\theta_{\text {in }}$, see Fig. 2 , causes an intensity response on the detector in $\left(\theta_{\text {out }}, d\right)$ space, where $\theta_{\text {out }}$ is the coordinate on the linear detector and $d$ is the delay in one interferometer arm. Note that the delay $d$ is not indicated in Fig. 2, nor the beam relay from entrance pupils to exit pupils. These intensity responses are available as analytical expressions, including the telescope diameter $D$, the wavelength $\lambda$, the telescope baseline $B_{\text {in }}^{(k)}$ for a series of $k=1,2, \ldots, K$ observations, the recombination baseline $B_{\text {out }}$ before the focusing optics, and the beam compression or angular magnification $M$. For the four interferometers treated here, these expressions are found from the general interferometer diffraction integral, according to

$$
\begin{aligned}
A^{(k)}\left(\theta_{\text {out }}, d\right) & \\
= & \int_{-(1 / 2) B_{\text {out }}-(1 / 2) D / M}^{-(1 / 2) B_{\text {out }}+(1 / 2) D / M} e^{i\left(2 \pi x \theta_{\text {out }} / \lambda\right)} M \\
& \times \exp \left\{i\left(2 \pi\left[-x M \theta_{\text {in }}+\frac{1}{2}\left(B_{\text {in }}^{(k)} / M-B_{\text {out }}\right) M \theta_{\text {in }}\right] / \lambda\right)\right\} \mathrm{d} x \\
& +e^{i(2 \pi d / \lambda)} \int_{(1 / 2) B_{\text {out }}-(1 / 2) D / M}^{(1 / 2) B_{\text {out }}+(1 / 2) D / M} e^{i\left(2 \pi x \theta_{\text {out }} / \lambda\right)} M \\
& \quad \times \exp \left\{i\left(2 \pi\left[-x M \theta_{\text {in }}-\frac{1}{2}\left(B_{\text {in }}^{(k)} / M-B_{\text {out }}\right) M \theta_{\text {in }}\right] / \lambda\right)\right\} \mathrm{d} x .
\end{aligned}
$$

The first exponential factor in the integrals constitutes the pupil-to-image relationship known from Fourier optics. The second exponential comprises the phases of secondary point sources in the exit apertures; the amplitudes of the secondary sources are scaled by a factor of $M$. For symmetry and ease of calculation, the term is primarily a function of $\theta_{\text {in }} x$ (the dashed wavefront in Fig. 2), to which a phase offset is added (the length of black arrows in Fig. 
2). Carrying out the integrations and using the definition of the sinc function,

$$
\operatorname{sinc}(x)= \begin{cases}1 & \text { for } x=0 \\ \frac{\sin (x)}{x}, & \text { otherwise }\end{cases}
$$

the integral in Eq. (1) results in $A^{(k)}\left(\theta_{\text {out }}, d\right)$ expressions that can be simplified and squared to arrive at an intensity response function for each specific interferometer.

A homothetic (HM) beam combiner has $B_{\text {out }}=B_{\text {in }}^{(k)} / M$ and produces a field-invariant point-spread function (PSF), given as

$$
\begin{aligned}
I_{k}\left(\theta_{\text {out }}\right)= & 4 D^{2} \operatorname{sinc}^{2}\left[\frac{D \pi}{M \lambda}\left(\theta_{\text {out }}-M \theta_{\text {in }}\right)\right] \\
& \times \cos ^{2}\left[\frac{B_{\text {in }}^{(k)} \pi}{M \lambda}\left(\theta_{\text {out }}-M \theta_{\text {in }}\right)\right],
\end{aligned}
$$

since the sinc envelope has the same origin as the cosinefringe function. Therefore, a polychromatic response [sums of Eq. (3) for several wavelengths $\lambda$ ] will show an envelope with a few fringes, where the central fringe will

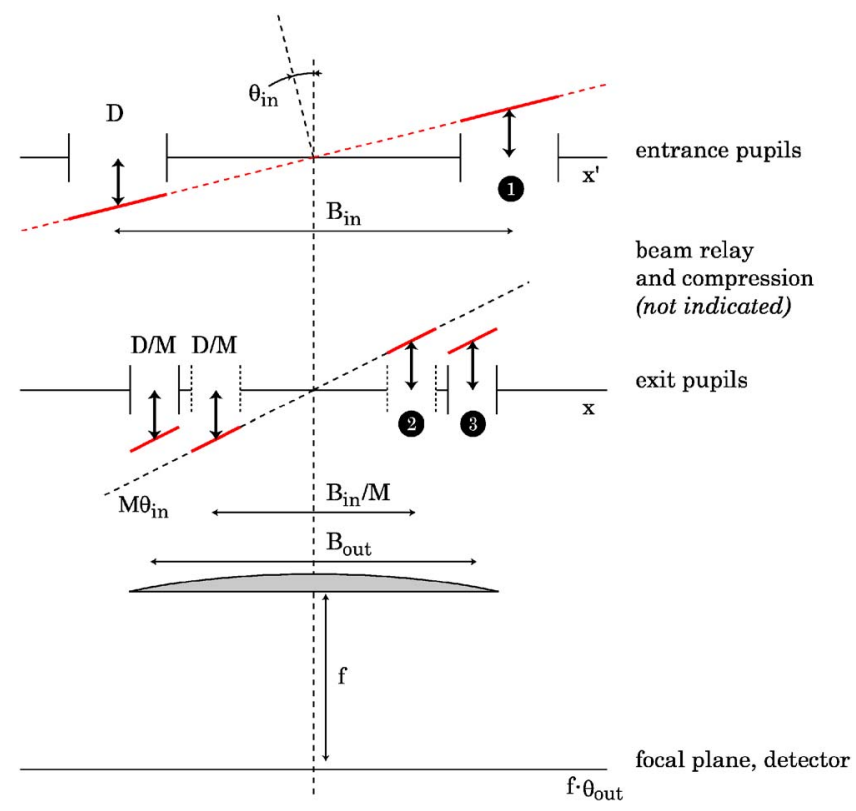

Fig. 2. (Color online) This illustration indicates the coordinates used in the derivation of the point-source response functions for all types of beam combination (any $B_{\text {out }} \geq 0$ ), when a general beam compression by a factor $M$ is applied in each arm. The diffraction integral is constructed symmetrically, leading to a compact expression. The phase differences at the collection plane $x^{\prime}$ are indicated with black arrows at the locations numbered 1, 2, and 3 . After beam compression, the phase slope in an aperture in the plane $x$ is proportional to $-x M \theta_{\text {in. }}$. The arrow at location 2 indicates that for proper exit pupil placement at a separation $B_{\text {in }}^{(k)} / M$, the light paths of a masked aperture are followed, since the wavefronts in the apertures are part of a single wavefront. Generally, see indicator 3, the aperture separation after beam relay is $B_{\text {out }}$, which is zero for coaxial beam combination. As a result, the wavefronts in the exit apertures, see indicator 3 , are parallel to the dotted line $-x M \theta_{\text {in }}$ but have an offset. As a result, a diffraction integral can be constructed, in which the phases proportional to $\left[-x M \theta_{\text {in }} \pm \frac{1}{2}\left(B_{\text {in }}^{(k)} / M-B_{\text {out }}\right) M \theta_{\text {in }}\right]$ occur. always be located centrally in the envelope. This property is lost for the densified beam combiner, where the response is

$$
\begin{aligned}
I_{k}\left(\theta_{\text {out }}, d\right)= & 4 D^{2} \operatorname{sinc}^{2}\left[\frac{D \pi}{M \lambda}\left(\theta_{\text {out }}-M \theta_{\text {in }}\right)\right] \\
& \times \cos ^{2}\left[\frac{\pi}{\lambda}\left(d+B_{\text {out }} \theta_{\text {out }}-B_{\text {in }}^{(k)} \theta_{\text {in }}\right)\right] .
\end{aligned}
$$

Only by making use of the internal delay $d$, the external delay $B_{\text {in }}^{(k)} \theta_{\text {in }}$ can be compensated. However, this compensation does not have to be perfect: for increasing values of $\theta_{\text {in }}$, the fringes drift off-center but are still visible. The magnification $M$ and the ratio $B_{\text {out }} / B_{\text {in }}^{(k)}$, or the densification factor, define how fast the central fringe drifts off, i.e., at what value of $\theta_{\text {in }}$ a jump in delay $d$ has to be made to place the fringes in the envelope again, as the sinc function has an off-axis origin at $M \theta_{\text {in }}$ while the cosine function originates at $B_{\text {in }}^{(k)} \theta_{\text {in }} / B_{\text {out }}$. As a result, for every measurement at $B_{\text {in }}^{(k)}$, a number of observations with different $d$ are to be made.

This is what interferometric imaging comes down to: when fringes can be measured, high-resolution imaging can be performed. The fringes have to cause intensity fluctuation in either the $\theta_{\text {out }}$ direction across the detector plane or in the $d$ direction or in both directions; detection of these intensities should take place at a number of locations in $\left(\theta_{\text {out }}, d\right)$ space. Since pixellation is as important as delay positions, considering a general interferometer, the intensity information is considered to be spread over detection bins.

The coaxial interferometers $\mathrm{MI}$ and staircase are described likewise. Since the recombination baseline $B_{\text {out }}$ is zero, Eq. (4) reduces to

$$
\begin{aligned}
I_{k}\left(\theta_{\text {out }}, d\right)= & 4 D^{2} \operatorname{sinc}^{2}\left[\frac{D \pi}{M \lambda}\left(\theta_{\text {out }}-M \theta_{\text {in }}\right)\right] \\
& \times \cos ^{2}\left[\frac{\pi}{\lambda}\left(d-B_{\text {in }}^{(k)} \theta_{\text {in }}\right)\right] .
\end{aligned}
$$

It is important to notice that the sinc function is still included in the response function. Although the fringes are no longer encoded spatially, an observation of a wide-field or extended source will need discrimination, on the incoherent- or low-spatial-frequency scale, of the recorded information with respect to the direction $\theta_{\text {in }}$. The required number of pixels in the $\theta_{\text {out }}$ direction is much lower than for the multiaxial interferometers. The coaxial response function in Eq. (5) shows a fringe function with an origin $B_{\text {in }}^{(k)} \theta_{\text {in }}$, meaning that for a polychromatic observation, the central fringe has moved far away from zero. Considering the general case of a large bandwidth and hence a few fringes present, this means that for an observation of a wide field of view (FOV), the detector has to be read out at many settings of $d$, ranging from $B_{\text {in }}^{(K)} \theta_{\text {in }}^{(\min )}$ to $B_{\text {in }}^{(K)} \theta_{\text {in }}^{(\max )}$, in steps of $\lambda_{c} / 4$. This large scanning range-or the narrow FOV when only a short classical scanning range of $-\lambda_{c}$ $<d<\lambda_{c}$ is considered-inspired the invention of external path-length compensation by means of a staircase-shaped mirror. ${ }^{6}$ The response function in this case transforms to 


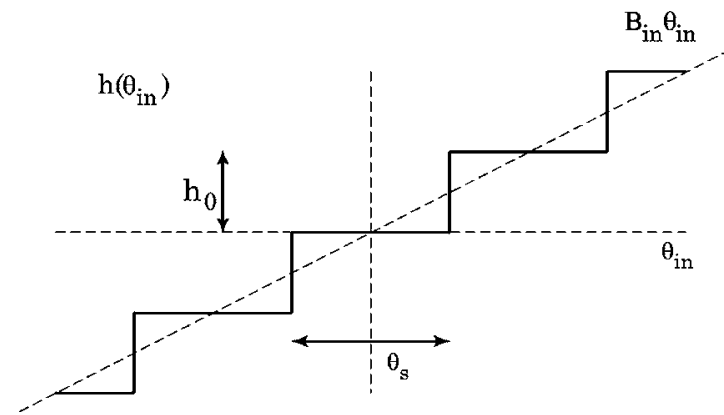

Fig. 3. Staircase function $h\left(\theta_{\text {in }}\right)$, as described in the text.

$$
\begin{aligned}
I_{k}\left(\theta_{\text {out }}, d\right)= & 4 D^{2} \operatorname{sinc}^{2}\left[\frac{D \pi}{M \lambda}\left(\theta_{\text {out }}-M \theta_{\text {in }}\right)\right] \\
& \times \cos ^{2}\left[\frac{\pi}{\lambda}\left(d-B_{\text {in }}^{(k)} \theta_{\text {in }}+h\left(\theta_{\text {in }}\right)\right)\right],
\end{aligned}
$$

with $h\left(\theta_{\text {in }}\right)$ as the staircase function, see Fig. 3. For a certain step height $h_{0}$ and step width $\theta_{s}$, the staircase function is given by

$$
\begin{aligned}
h\left(\theta_{\text {in }}\right)= & \sum_{n=-\infty}^{+\infty} n h_{0} \operatorname{rect}\left[\frac{\theta_{\text {in }}-n \theta_{s}}{\theta_{s}}\right] \\
= & \sum_{n=-\infty}^{+\infty} n h_{0}\left[H\left\{\frac{\theta_{\text {in }}-\left(n-\frac{1}{2}\right) \theta_{s}}{\theta_{s}}\right\}\right. \\
& \left.-H\left\{\frac{\theta_{\text {in }}-\left(n+\frac{1}{2}\right) \theta_{s}}{\theta_{s}}\right\}\right],
\end{aligned}
$$

with $n \in \mathbb{Z}$. Here, the Heaviside step function $H(x)$ is used, given as

$$
H(x)=\left\{\begin{array}{ll}
0, & x<0 \\
\frac{1}{2}, & x=0 \\
1, & x>0
\end{array} .\right.
$$

The step width $\theta_{s}$ and step height $h$ have to correspond and need to be altered when changing the baseline length $B_{\text {in }}^{(k)}$ according to $h_{0}=B_{\text {in }}^{(k)} \theta_{s}$ or $\theta_{s}=h_{0} / B_{\text {in }}^{(k)}$, to equalize the external optical path-length difference over the complete FOV.

Now that we have specified the point-source response for four types of interferometers, the actual imaging of a stellar source can be considered. A stellar source is regarded to be a collection of incoherent point sources of which the intensity responses can be summed. Another example of treating partially coherent imaging in this way can be found in a paper by Young and Hale. ${ }^{11}$ The sum of intensity responses can be performed after collecting all possible point-source responses in a transfer matrix.

\section{B. Interferometric Transfer Matrix}

To construct a transfer matrix, an observational layout has to be defined, specifying either the desired resolution of the obtained image or the constraints on the detection side, when, for example, the scanning range in $d$ is lim- ited or the detector pixels are limited in size. In all cases, the source grid and detection grid have to be discretized according to

$$
\begin{aligned}
\boldsymbol{\theta}_{\text {in }} & =\left(\theta_{\text {in }}^{(1)}, \theta_{\text {in }}^{(2)}, \ldots, \theta_{\text {in }}^{(n)}, \ldots, \theta_{\text {in }}^{(N)}\right), \\
\mathbf{d} & =\left(d_{1}, d_{2}, \ldots, d_{p}, \ldots, d_{P}\right), \\
\boldsymbol{\theta}_{\text {out }} & =\left(\theta_{\text {out }}^{(1)}, \theta_{\text {out }}^{(2)}, \ldots, \theta_{\text {out }}^{(j)}, \ldots, \theta_{\text {out }}^{(J)}\right)
\end{aligned}
$$

for every baseline length $B_{\text {in }}^{(k)}$, with $k=1,2, \ldots, K$. Then, the transfer of a stellar source, expressed as a luminous intensity function $L\left(\theta_{\text {in }}\right)$, is related to a set of $K$ intensity patterns, stored per pixel as $I_{j k}(d)$. If the forward problem is denoted with operator $g$ as

$$
I_{j k}\left(d_{p}\right)=g\left[L\left(\theta_{\text {in }}\right), B_{\text {in }}^{(k)}, \theta_{\text {out }}^{(j)}\right],
$$

then the inverse problem is found by $g^{-1}$ in

$$
\hat{L}\left(\theta_{\text {in }}\right)=g^{-1}\left[I_{j k}\left(d_{p}\right)\right]
$$

so that the luminous intensity function $\hat{L}\left(\theta_{\text {in }}\right)$ can be estimated from the measured series $I_{j k}\left(d_{p}\right)$ given the parameters $B_{\text {in }}^{(k)}$ and $\theta_{\text {out }}^{(j)}$, the lengths of the baselines at which the data were taken, and the central locations of the pixels. Instead of using operator $g$, the forward problem can be denoted as a matrix-vector multiplication $\mathbf{A x}=\mathbf{b}$, where the discrete vector $\mathbf{x}$ is a $n \times 1$ source function $L\left(\theta_{\text {in }}\right)$. The measurement is stored in vector $\mathbf{b}$, of size $(J P K) \times 1$ and is constructed as

$$
\mathbf{b}=\left(\begin{array}{c}
I_{j 1}(\mathbf{d}) \\
I_{j 2}(\mathbf{d}) \\
\ldots \\
I_{j k}(\mathbf{d})
\end{array}\right),
$$

and the $I_{j k}(\mathbf{d})$ are column vectors of length jp. The matrix A is sized $(J P K) \times N$ and is actually a collection of $\mathbf{b}$ column vectors, giving the response when source vectors $\mathbf{x}$ are taken that are zero except for one element per vector. As such, matrix $\mathbf{A}$ is the transfer matrix of the interferometric imaging system, relating a collection of independent point sources $L\left(\theta_{\text {in }}\right)$ to a summed intensity pattern in $\left(\theta_{\text {out }}, d\right)$ space, for a number of baselines. The columns of A resemble their neighboring columns, since, e.g., in the coaxial case for a small grid spacing $\Delta \theta_{\text {in }}=\left(\theta_{\text {in }}^{(2)}-\theta_{\text {in }}^{(1)}\right)$, the fringe shift or external delay $B_{\text {in }}^{(k)} \Delta \theta_{\text {in }}$ can be small, resulting in nearly equal columns. Physically, this means that a recorded fringe function is hard to relate to a specific source direction. As a result, matrix $\mathbf{A}$ has a large condition number, and the problem is ill-conditioned or illposed. Nonetheless, matrices can be constructed for all interferometers, and they contain all necessary information to compare the imaging performance of the interferometers.

\section{Detection Parameters}

The values $J, P, K$, and $N$ are chosen as follows. The desired FOV and synthetic resolution can freely be chosen; the analysis of the transfer matrix will show if the desired resolution is achieved in the full FOV, or whether the cho- 
sen spacing is too small. The grid of the $\theta_{\text {in }}$ values does not have to be equidistant, but in these examples it will be. The number of baselines also relates to the resolution: the image information of ultimate resolution is added when fringes are measured at the maximum value of the chosen baseline lengths; the angular resolution will be $\sim \lambda_{c} / B_{\text {in,max }}$, much higher than the incoherent resolution $\left(\sim \lambda_{c} / D\right)$ of a single aperture. After fixing the maximum baseline length in accordance with the desired resolution, the number of baselines is simply $B_{\text {in,max }} / D$, since to obtain an image containing all spatial frequencies, the synthetic aperture will have to be filled in completely. For polychromatic observations with monochromatic (luminous intensity) image reconstructions, larger steps in $B_{\text {in }}^{(k)}$ are allowed, since the wavelength range causes overlap of spatial frequencies when sampling at, e.g., $B_{\text {in }}^{(k)}$ and $B_{\text {in }}^{(k+1)}$.

The number of delay steps $p$ is such that fringes in $d$ direction (for the coaxial methods) are at least sampled with $\lambda_{c} / 4$ intervals, and that the $d$ positions of the central fringes of point sources at the outer edges of the FOV are reached with a delay scan, causing a scanning range of $\theta_{\text {in }}^{(\min )} B_{\text {in,max }}<d<\theta_{\text {in }}^{\text {max }} B_{\text {in,max }}$ for the MI case and $-h_{K}<d$ $<h_{K}$ for the case of staircase compensation, where $h_{K}$ is the maximum stair height.

The angular pixel dimensions in the focal plane are for the coaxial imagers half the angular size of the incoherent diffraction spot $\left(\sim \frac{1}{2} \lambda_{c} / D\right)$ and for the multiaxial imagers a quarter of the angular width of a fringe $\left(\sim \frac{1}{4} \lambda_{c} / B_{\text {out }}^{\text {max }}\right)$, in order to sample each fringe again with four samples. For densified pupil imaging, $B_{\text {out }}$ is a fixed value causing the same number of samples per fringe for each value $B_{\text {in }}^{(k)}$; for $\mathrm{HM}$ imaging, the number of pixels on the detector array is essentially chosen such that the ultimate spatial resolution on the sky can be sampled. All snapshots taken at shorter baselines are oversampled. The characteristics of the four interferometers are listed in Table 1. For the

\section{Table 1. Characterization of the Beam Combiner Optics in the Interferometers under Consideration $^{a}$}

\begin{tabular}{lcccc}
\hline Property & HM & Densified & MI & Staircase \\
\hline Axiality & Multi & Multi & Coaxial & Coaxial \\
Recombination & $B_{\text {out }}=B_{\text {in }}^{(k)} / M$ & $B_{\text {out }}=2 D$ & $B_{\text {out }}=0$ & $B_{\text {out }}=0$ \\
Magnification & $M=1$ & $M=1$ & $M=1$ & $M=1$ \\
Fringe encoding & $\theta_{\text {out }}$ & $\theta_{\text {out }}, d$ & $d$ & $d$ \\
\hline
\end{tabular}

${ }^{a}$ The magnification was chosen to be $M=1$ for simplicity. simulation runs with the four methods, the corresponding discretizations of the $\left(\theta_{\text {out }}, d\right)$-observation spaces are given in Table 2.

The telescope diameter is in all cases $D=3.5 \mathrm{~m}$, and the observation is performed for a large set of wavelengths $\lambda$ ranging from 6 to $10 \mu \mathrm{m}$. The polychromatic response is obtained by summing the intensity signals for each wavelength. This leads to a coherence length $l_{\text {coh }}=\lambda_{c}\left[\lambda_{c} /\left(\lambda_{\max }\right.\right.$ $\left.\left.-\lambda_{\min }\right)\right]$ of $16 \mu \mathrm{m}$. The baseline lengths $B_{\text {in }}^{(k)}$ at which the snapshots are taken, are equal for all interferometers and given as

$$
B_{\text {in }}^{(k)}= \begin{cases}2 D, & k=1 \\ 4(k-1) D, & k=2,3, \ldots, 9\end{cases}
$$

The baseline length differences of $4 D$ might seem large, but with an observational bandwidth for which $6 \mu \mathrm{m}$ $\leq \lambda_{c} \leq 10 \mu \mathrm{m}$, this set of baselines leads to a spatialfrequency coverage with no gaps up to the desired ultimate spatial frequency.

Table 2 provides a list of the detector discretizations, needed for the four different imagers, in order to cover the FOV and reach the same spatial-angular-frequency coverage. For the methods other than densified, the discretization is straightforward. The necessary range of delay steps for the densified case to have coherent information from all regions of the sky is derived from the modulation part of the intensity point-source response for a general interferometer, given as

$$
\cos \left[\frac{\pi B_{\text {out }}}{\lambda}\left(\theta_{\text {out }}-\left[\frac{B_{\text {in }}}{B_{\text {out }}} \theta_{\text {in }}\right]+\frac{d}{B_{\text {out }}}\right)\right]=1 \quad \text { for } \theta_{\text {in }}=\theta_{\text {in }}^{(\max )} \text {. }
$$

The expression of Eq. (14) states that a bright fringe is necessary at the boundary of the FOV. Since the observation is polychromatic and only a few fringes will be present, this requirement is reduced to finding the delay $d$, for which the central fringe appears in the center of the diffraction envelope. For the edge of the FOV at magnification $M=1$, this center is located at $\theta_{\text {out }}=\theta_{\text {in }}^{(\max )}$. Equation (14) now reduces to

$$
d=B_{\text {out }} \theta_{\text {in }}\left(1-\frac{B_{\text {in }}}{B_{\text {out }}}\right),
$$

from which discrete settings of $d$ are derived; three in this case. These three settings make that for the observations

\begin{tabular}{|c|c|c|c|c|}
\hline Parameter & $\mathrm{HM}$ & Densified & MI & Staircase \\
\hline Detector size & FOV & FOV & FOV & FOV \\
\hline Pixel size & $p_{\theta}\left(B_{\text {out,max }}\right) / 4$ & $p_{\theta}\left(B_{\text {out }}\right) / 4$ & $p_{\theta}(D) / 2$ & $p_{\theta}(D) / 2$ \\
\hline Number of pixels & 235 & 31 & 6 & 6 \\
\hline Scanning range & - & $-\frac{3}{2} l_{\mathrm{coh}} \cdots+\frac{3}{2} l_{\mathrm{coh}}$ & $2 B_{\text {in,max }} \theta_{\text {in }}^{(\max )}$ & $2 l_{\mathrm{coh}}$ \\
\hline Delay step size & - & $l_{\mathrm{coh}}$ & $\lambda_{c} / 4$ & $\lambda_{c} / 4$ \\
\hline Number of steps & 1 & 3 & 234 & 25 \\
\hline Bins total & 235 & 93 & 1404 & 150 \\
\hline
\end{tabular}
at $B_{\text {in,max }}$ fringed PSFs can be observed in the right-hand

Table 2. Discretization of the $\left(\theta_{\text {out }}, d\right)$ Detection Space for the Simulations ${ }^{a}$

${ }^{a}$ The FOV is related to the size of the incoherent diffraction limit for an aperture of diameter $D$ and equals FOV $=3 \mathrm{PSF}$, where PSF $=1.22 \lambda_{c} / D$. The angular fringe period in focus $p_{\theta}$ is derived from the general intensity response function and equals $p_{\theta}\left(B_{\text {out }}\right)=\lambda_{c} / B_{\text {out }}$. 
side, the central, and the lefthand side regions of the sky for the respective setting of $d$.

\section{SINGULAR VALUE DECOMPOSITION}

\section{A. Ill-Posed Problems}

The generalized and discrete imaging equation

$$
\mathbf{b}=\mathbf{A} \cdot \mathbf{x},
$$

in itself is an illustrative construction of measurement signals, giving insight into the partially coherent nature of this type of observation. Intensity patterns from individually incoherent but hardly separated point sources are summed, resulting in an intensity response that depends on the type of interferometer and particular settings such as the telescope separation $B_{\text {in }}^{(k)}$. The imaging equation however, was actually constructed to obtain estimates of $\hat{L}\left(\theta_{\text {in }}\right)$ expressed as $\hat{\mathbf{x}}$ out of measured and hence noisy $\mathbf{b}$. Without wanting to deal with the measurement procedure, we will show that, first, inversion of the matrix-vector approach to interferometric imaging is possible and, second, that the solution of this inversion problem gives additional insight into the imaging problem.

A naive reconstruction $\hat{L}\left(\theta_{\text {in }}\right)$ of an original source $L\left(\theta_{\text {in }}\right)$ with average amplitude 1 results from the MoorePenrose inverse $\hat{\mathbf{x}}=\left(\mathbf{A}^{T} \mathbf{A}\right)^{-1} \mathbf{A}^{T} \cdot \mathbf{b}$ with added noise to $\mathbf{b}$. The result is a seemingly random estimate of very high magnitude $\left(10^{4}\right.$ instead of the expected 1$)$, with a large number of sign changes, see Fig. 4 . This behavior is typical for inversion of ill-posed or ill-conditioned problems. Clearly, a different approach is necessary to derive meaningful solutions to the inverse imaging equation.

\section{B. Analysis}

A particularly useful numerical tool for the analysis of illconditioned problems is the singular value decomposition (SVD) since it reveals all the difficulties associated with the ill-conditioning of matrix $\mathbf{A} .^{12,13}$ Considering the rectangular transfer matrix $\mathbf{A} \in \mathbb{R}^{m \times n}$, the SVD of this matrix is written as

$$
\mathbf{A}=\mathbf{U} \cdot \mathbf{S} \cdot \mathbf{V}^{T}=\sum_{i} \mathbf{u}_{i} \sigma_{i} \mathbf{v}_{i}^{T}
$$

In the case of an overdetermined system $(m>n)$, matrix $\mathbf{U}$ is of dimensions $m \times n$, and $\mathbf{V}$ is of $n \times n$. For an underdetermined system $(m<n)$, $\mathbf{U}$ is of dimensions $m \times m$, and $\mathbf{V}$ is of $n \times m$. In both cases, $\mathbf{U}$ and $\mathbf{V}$ are unitary matrices- $\mathbf{U}^{T} \cdot \mathbf{U}=\mathbf{I}_{m}$ and $\mathbf{V}^{T} \cdot \mathbf{V}=\mathbf{I}_{n}$-with their columns representing the left and right singular vectors $\mathbf{u}_{i}$ and $\mathbf{v}_{i}$ :

$$
\mathbf{U}=\left[\mathbf{u}_{1} \mathbf{u}_{2} \cdots \mathbf{u}_{\min (m, n)}\right], \quad \mathbf{V}=\left[\mathbf{v}_{1} \mathbf{v}_{2} \cdots \mathbf{v}_{\min (m, n)}\right] .
$$

The nonnegative and real singular values $(\mathrm{SVs}) \sigma_{i}$ are collected on the diagonal of matrix $\mathbf{S}=\operatorname{diag}\left(\sigma_{1} \sigma_{2} \cdots \sigma_{\min (m, n)}\right)$ in descending order such that $\sigma_{1} \geq \sigma_{2} \geq \cdots \geq \sigma_{\min (m, n)} \geq 0$.

The condition number of $\mathbf{A}$ equals the ratio of the largest and smallest SV:

$$
\operatorname{cond}(\mathbf{A})=\sigma_{1} / \sigma_{\min (m, n)} .
$$

This ratio is a measure for the sensitivity of the solution to perturbation errors in matrix $\mathbf{A}$ or the right-hand side
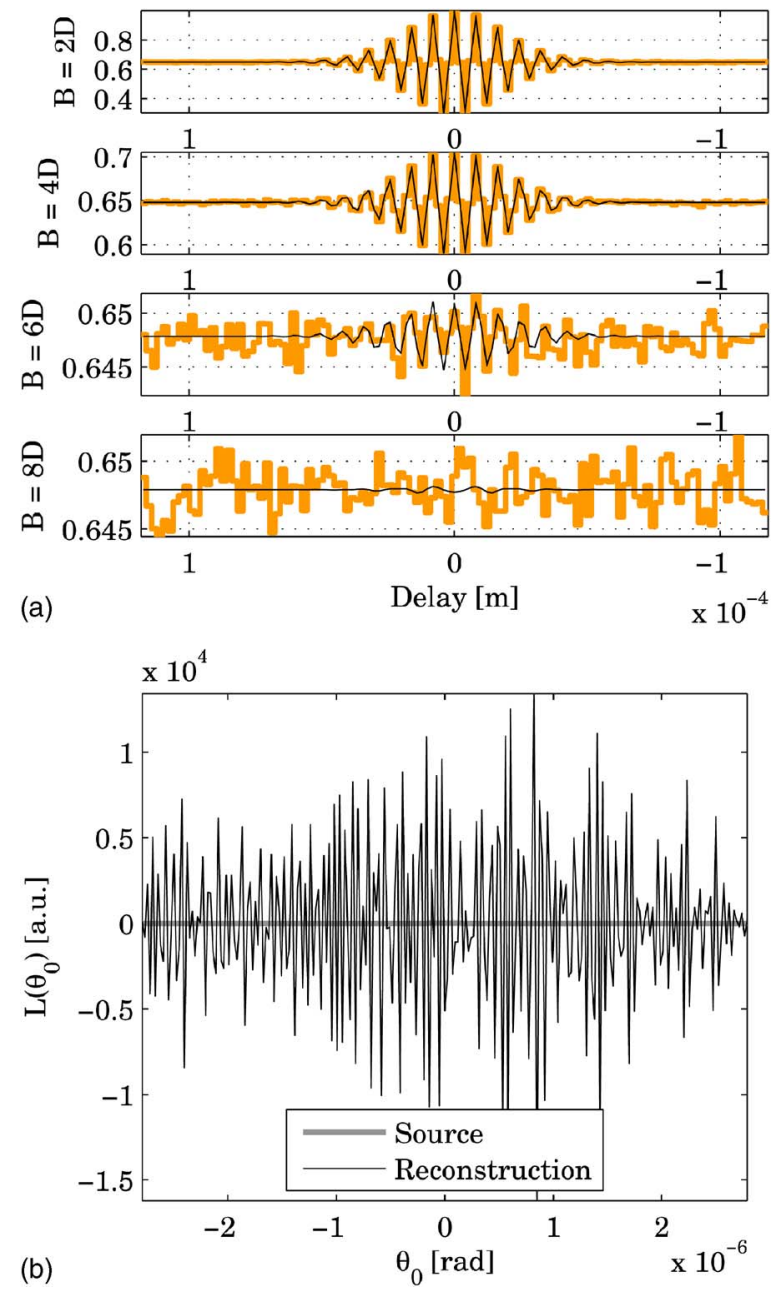

Fig. 4. (Color online) Naïve inversion of interferometric data. Panel (a) shows a reorganized measurement vector $\overline{\mathbf{b}}$, resembling intensity interferograms recorded at four different baseline lengths, obtained as $\overline{\mathbf{b}}=\mathbf{A} \cdot \mathbf{x}$, where $\mathbf{x}$ is a positive source function $L\left(\theta_{\text {in }}\right)$ with maximum amplitude 1 in arbitrary units (arb. units). The results are then photon quantized, resulting in $\mathbf{b}=\overline{\mathbf{b}}$ $+\mathbf{e}_{b}$. Panel (b) then shows the naive reconstruction $\hat{\mathbf{x}}$ $=\left(\mathbf{A}^{T} \mathbf{A}\right)^{-1} \mathbf{A}^{T} \cdot \mathbf{b}$, where the pseudoinverse or Moore-Penrose inverse is used since $\mathbf{A}$ is not square. The result is an estimate $\hat{L}\left(\theta_{\text {in }}\right)$ of very high magnitude ( $10^{4}$ instead of 1 for the source), with a large number of sign changes. Note the scale of the vertical axis causing the source to appear as a straight line at zero.

b. It has to be remarked that the SVD presented in Eq. (17) is a so-called economy-sized type of decomposition, i.e., the SVs and vectors associated with the null space of matrix $\mathbf{A}$ are intentionally left out of consideration to reduce the computational cost of this calculation. In connection with discrete ill-posed problems, three characteristic features of the SVD of $\mathbf{A}$ are often found ${ }^{13-16}$ :

1. The $\mathrm{SVs} \sigma_{i}$ decay gradually to zero with no particular gap in the spectrum.

2. The condition number of $\mathbf{A}$ is large.

3. The left and right singular vectors $\mathbf{u}_{i}$ and $\mathbf{v}_{i}$ tend to have more sign changes in their elements as index $i$ increases. In other words, for the lower values of $\sigma_{i}$, the singular vectors become more oscillatory. 
These characteristics are confirmed by the decomposition of the imaging transfer matrix, e.g., the multiple baseline HM interferometer transfer matrix. Figure 5 clearly illustrates the gradual decay of the SVs $\sigma_{i}$ (item 1) and the large condition number (item 2), spanning many orders of magnitude. In the figure, two lines separate the range of indices $i$ into three regimes. The regimes and the oscillatory behavior (item 3) are illustrated in Fig. 6. It is confirmed that the left- and right-hand singular vectors indeed contain more oscillations as the index of the SV increases. Further observation of these vectors leads to a description of three regimes, physically relating to the fact that the vectors result from an interferometric imaging matrix. The modes and magnitudes of the corresponding $\mathrm{SVs}$ can be separated into three regimes, based on their index $i$ :

- Incoherent regime: For indices $1 \leq i \leq n_{\mathrm{PSFs}}$, where $n_{\mathrm{PSF}}$ is the number of incoherent PSFs fitting in the FOV, the modes relate to the low-resolution or incoherent image of the sky.

- Interferometric regime: The modes for indices $n_{\mathrm{PSFs}}$ $<i<i_{B}$, where the major spatial frequency of the mode with index $i_{B}$ relates to the maximum baseline length $B_{\text {in,max }}$, are the modes related to the interferometric character of the array.

- Unsampled regime: The region of indices $i>i_{B}$ contains modes of very high spatial frequency, which do not physically relate to the source or the measurement, since these spatial frequencies are not sampled. This occurs when the discretization step size $\left(\theta_{\text {in }}^{(2)}-\theta_{\text {in }}^{(1)}\right)$ is smaller than the smallest spatial period that is sampled, $\lambda_{\min } / B_{\text {in,max }}$.

Clearly, the latter set of modes (related to the smallest SVs $\sigma_{i}$ ) has to be banned in all cases from the construction of a solution to the inverse problem.

The SVD also gives important insight into another aspect of ill-conditioned problems, namely, the smoothing effect. This is typically associated with integral kernels encountered in many physical problems. ${ }^{13,15}$ For example, consider the mathematical mapping $\mathbf{b}=\mathbf{A} \cdot \mathbf{x}$ of an arbitrary vector $\mathbf{x}$. Application of the SVD yields

$$
\mathbf{b}=\mathbf{U} \cdot \mathbf{S} \cdot \mathbf{V}^{T} \cdot \mathbf{x}, \quad \text { or } \quad \mathbf{b}=\sum_{i} \sigma_{i}\left(\mathbf{v}_{i}^{T} \cdot \mathbf{x}\right) \mathbf{u}_{i} .
$$

The product $\mathbf{v}_{i}^{T} \cdot \mathbf{x}$ can be interpreted as a measure for the spatial matching (or participation factor) between singu-

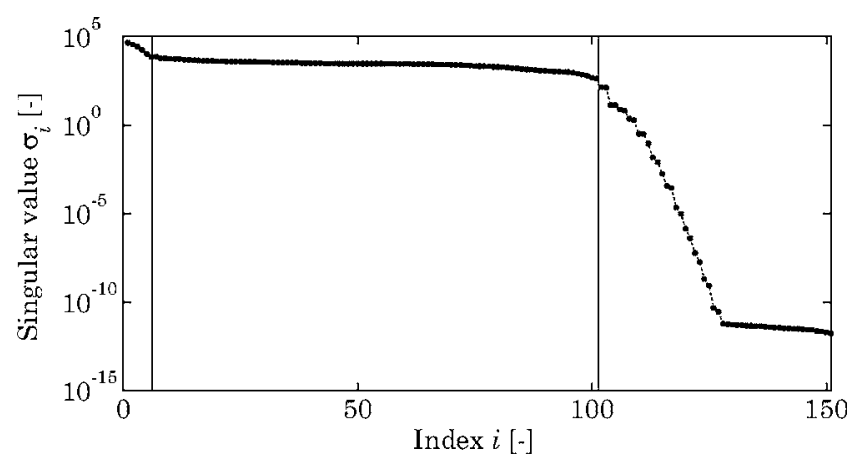

Fig. 5. Singular value spectrum of a $1592 \times 151$ image transfer matrix $\mathbf{A}$, as in Eq. (16). The condition number is $\operatorname{cond}(\mathbf{A})=2.40$ $\times 10^{16}$.

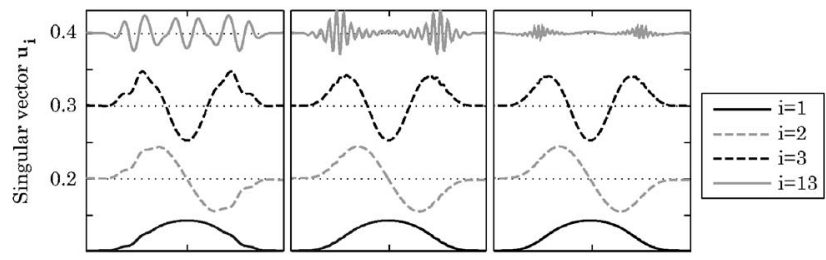

Detector position $\theta_{\mathrm{out}}$ for $B_{k}, k=1,3,5[-]$

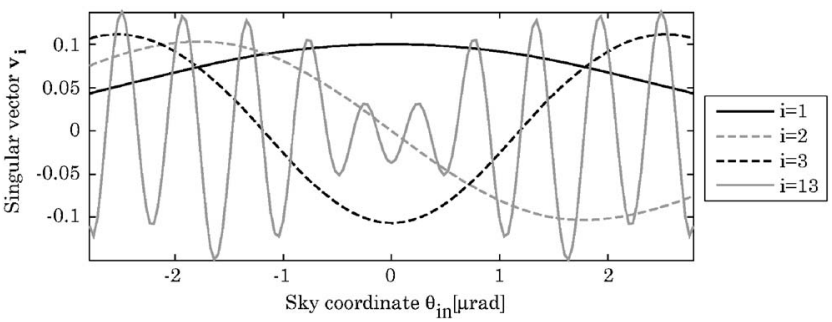

Fig. 6. Singular vectors show more oscillations for higher index $i$. Vectors $\mathbf{u}_{i}$ can be interpreted as measurement modes whereas vectors $\mathbf{v}_{i}$ represent source modes. The vectors $\mathbf{u}_{i}$ are plotted with offsets in the vertical direction, and only for three baselines $B_{\text {in }}^{(k)}$, where $k=1,3,5$.

lar vector $\mathbf{v}_{i}$ and vector $\mathbf{x}$. With this knowledge and recalling that the singular vectors $\mathbf{u}_{i}$ and $\mathbf{v}_{i}$ become more oscillatory as $\sigma_{i}$ decreases, it is obvious that the multiplication with $\sigma_{i}$ leads to more damping of the high-frequency components of $\mathbf{x}$ and less damping of the low-frequency components in the map $\mathbf{A} \cdot \mathbf{x}$. As a consequence, in forward problems, the solution $\mathbf{b}$ is generally smoother than the prescribed vector $\mathbf{x}$.

In contrast, the inverse problem (containing $1 / \sigma_{i}$ ) will show the opposite effect, amplifying the high-frequency oscillations in the right-hand side b. Owing to the smoothing characteristics of the forward problem, noise often has a relatively strong contribution to the highfrequency oscillations in $\mathbf{b}$. Therefore, especially the noisy components are subjected to a large amplification in the inverse process.

Concluding, the forward operation of mapping input vector $\mathbf{x}$ onto output vector $\mathbf{b}$ has a smoothing effect on $\mathbf{b}$, whereas the opposite operation tends to amplify oscillations in vector $\mathbf{b}$. Hence, if a solution of $\mathbf{x}$ is required with a limited $L_{2}$ norm, then not all distributions $\mathbf{b}$ form a valid right-hand side. In fact, vector $\mathbf{b}$ must be sufficiently smooth to survive the inversion back to a physically meaningful $\mathbf{x}$.

Naively solving the inverse problem results in constructing the least-squares solution. With the SVD as defined in Eq. (17), this solution can be written as

$$
\mathbf{x}_{\mathrm{LS}}=\sum_{i} \frac{\mathbf{u}_{i}^{T} \cdot \mathbf{b}}{\sigma_{i}} \mathbf{v}_{i}
$$

where the factors $\mathbf{u}_{i}^{T} \cdot \mathbf{b} / \sigma_{i}$ represent the contribution of each singular vector $\mathbf{v}_{i}$ (source mode) in the least-squares solution $\mathbf{x}_{\mathrm{LS}}$. If now the Fourier components $\left|\mathbf{u}_{i}^{T} \cdot \mathbf{b}\right|$ are leveled off at a noise floor, they cannot decay as fast as the $\mathrm{SVs} \sigma_{i}$. The direct consequence is that the solution $\mathbf{x}_{\mathrm{LS}}$ is dominated by terms in the sum corresponding to the smallest $\mathrm{SVs} \sigma_{i}$; thus, the shape of the solution is mainly determined by the source mode shapes $\mathbf{v}_{i}$ associated with large $i$. Since these mode shapes have a highly oscillatory 
nature, the solution appears to be completely random. Apart from the many oscillations, the magnitude of the solution $\mathbf{x}_{\mathrm{LS}}$ usually ends up being extremely large owing to the division by the very small SVs. This knowledge clarifies that the purpose of regularization is to dampen or filter out the contributions to the solution corresponding to the small SVs.

\section{TIKHONOV REGULARIZATION}

Once it is known that the ill-behavior is related to the smallest SVs $\sigma_{i}$, the most obvious cure is to simply truncate the number of terms in the solution $\mathbf{x}_{k}$ :

$$
\mathbf{x}_{k}=\sum_{i=1}^{k} \frac{\mathbf{u}_{i}^{T} \cdot \mathbf{b}}{\sigma_{i}} \mathbf{v}_{i}, \quad \text { with } k \leq \min (m, n) .
$$

This truncation effectively eliminates the influence of all singular vectors $\mathbf{v}_{i}$ associated with SVs smaller than $\sigma_{k}$. Essentially, the truncated singular value decomposition (TSVD) approach replaces the ill-conditioned transfer matrix $\mathbf{A}$ with a new well-conditioned, but rank deficient, matrix $\mathbf{A}_{k}$ (as described by Hansen ${ }^{15}$ ). The participation of modes $\mathbf{v}_{i}$ in the solution is either $\mathbf{u}_{i}^{T} \mathbf{b} / \sigma_{i}$, or zero. A method to limit the participation of the higher-order modes in a more subtle way is Tikhonov regularization.

Tikhonov regularization ${ }^{12}$ is probably the most popular and well-developed method to handle discrete ill-posed problems. It differs from the TSVD approach in the fact that the initial ill-conditioned problem is solved simultaneously with a side constraint imposed on certain properties of the sought solution. ${ }^{13}$ These constraints are usually associated with smoothness properties of the solution since ignoring contributions related to small SVs leads to a smoother solution with a reasonable $L_{2}$ norm. In discrete problems, the problem of Tikhonov regularization takes the form:

$$
\min _{\mathbf{x}}\left\{\|\mathbf{A} \cdot \mathbf{x}-\mathbf{b}\|^{2}+\Lambda^{2}\|\mathbf{L} \cdot \mathbf{x}\|^{2}\right\}
$$

where $\mathbf{L}$ represents the so-called regularization matrix and $\Lambda$ is the regularization parameter. For a discussion of choosing the smoothing operator $\mathbf{L}$, the reader is referred to the work by Visser ${ }^{16}$ on acoustical transfer matrices.

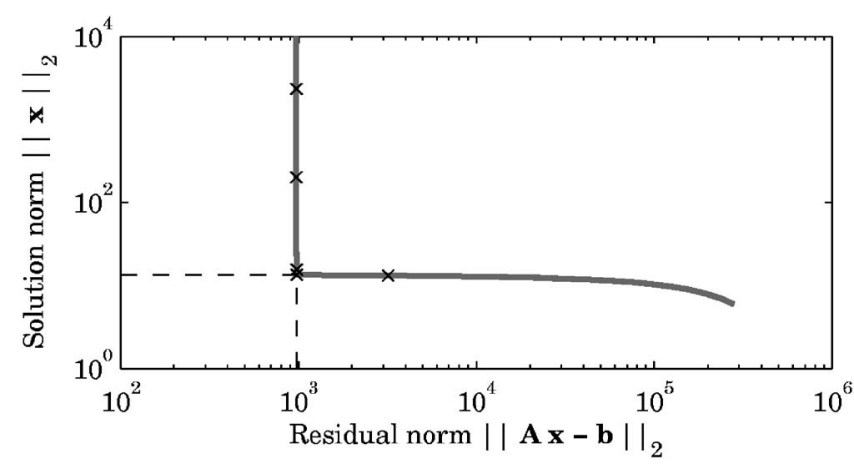

Fig. 7. L curve is a method to blindly find the optimum regularization parameter $\Lambda$. The norms $\left\|\mathbf{A} \cdot \mathbf{x}_{\Lambda}-\mathbf{b}\right\|$ and $\left\|\mathbf{x}_{\Lambda}\right\|$ are plotted for a range of $\Lambda$ values. The corner is found as the minimum radius of curvature in a spline fit through the calculated points. The standard two norms are taken.

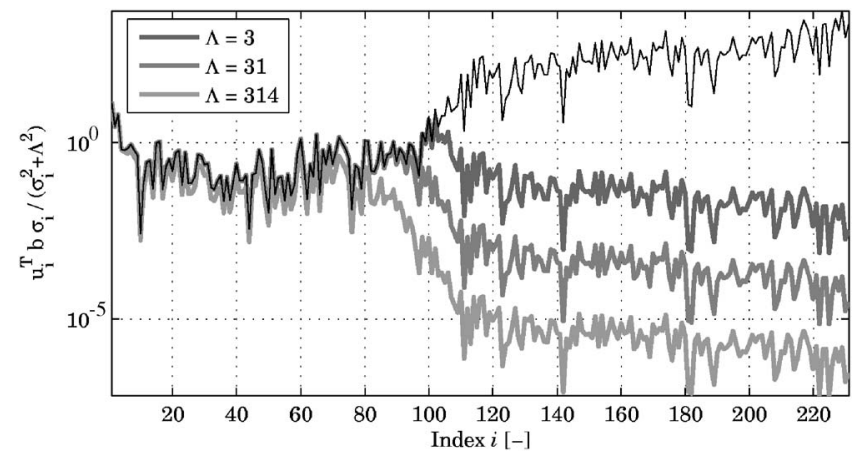

Fig. 8. Plot of the mode participation, expressed as $\mathbf{u}_{i}^{T} \mathbf{b} / \sigma_{i}$, for a mode with index $i$. The thin curve (no regularization, $\Lambda=0$ ) shows that indeed the participation of noise-sensitive, higher index modes is strong. The thick curves show the regularized mode participation for $\Lambda=\Lambda_{\text {opt }}\left(\frac{1}{10}, 1,10\right)$.

The most simple form of Eq. (23) is obtained with $\mathbf{L}$ $=\mathbf{I}$. The Tikhonov regularized solution $\mathbf{x}_{\Lambda}$ can in combination with the SVD be written as

$$
\mathbf{x}_{\Lambda}=\sum_{i} \frac{\mathbf{u}_{i}^{T} \cdot \mathbf{b} \sigma_{i}}{\sigma_{i}^{2}+\Lambda^{2}} \mathbf{v}_{i}
$$

The regularization parameter $\Lambda$ is chosen to lie between the largest and the smallest SVs. Like the TSVD approach, the Tikhonov method acts as a low-pass filter in the SV spectrum, be it that TSVD manifests an abrupt cutoff, whereas the filter spectrum in the Tikhonov case shows a gradual decline. Regularization of a linear inverse problem now comes down to finding the regularization parameter $\Lambda$, so that the norms

$$
\left\|\mathbf{A} \cdot \mathbf{x}_{\Lambda}-\mathbf{b}\right\|, \quad\left\|\mathbf{x}_{\Lambda}\right\|
$$

respectively, the data misfit norm and the solution norm, are small. Figure 7 plots these two norms against each other, for a range of values of $\Lambda$. The result is a plot that clearly has an L shape, as is generally the case for illposed discrete inverse problems. ${ }^{15}$ In the corner, the sum of the two norms is smallest, meaning that the corresponding $\Lambda$ minimizes the expression in Eq. (23).

As $\Lambda$ is found, a solution can be calculated with Eq. (24). The participation of the solution modes $\mathbf{v}_{i}$ can now be controlled. Figure 8 shows the participation strength $\mathbf{u}_{i}^{T} \cdot \mathbf{b} \sigma_{i} /\left(\sigma_{i}^{2}+\Lambda^{2}\right)$ for three increasing values of $\Lambda$, corresponding to an underregularized solution, an optimally regularized solution, and an overregularized solution. In Fig. 9, the corresponding regularized solutions are plotted. These show an estimate with amplification of noise (underregularization), an optimal estimate and an estimate, unable to recover sharp edges (oversmoothing due to overregularization).

\section{DARWIN OPTIONS COMPARED}

We have described the simulation of an interferometric observation, which consists in: generating response functions according to the chosen beam combiner and array 
configurations, summing these to a measured intensity signal, perturbing that signal to represent low-light levels and imperfect detection and, finally, estimating an image out of these signals. The sources of noise are not specified in this paper-the described regularization procedure can be assumed to be able to deal with any degradation of the measurement signals $\mathbf{b}$ or perturbations in the matrix $\mathbf{A}$ itself. Based on the generated estimations, a comparison of the imaging performance of the interferometers under consideration, based on different recombination schemes and detectors, can be made. In this section, we compare the imaging performance of the interferometers solely on the basis of the response functions and transfer matrices. With the transfer matrices available, a plot can be made of the SVs related to the four interferometers and the chosen array parameters, such as delay settings and pixel dimensions.

The general regularization process for a discrete illposed inverse problem has been described in Section 4. For the case of interferometry, out of a measurement vector $\mathbf{b}$, an estimate of the source $\hat{L}\left(\theta_{\text {in }}\right)$, represented by vector $\mathbf{x}_{\Lambda}$, is given according to

$$
\mathbf{x}_{\Lambda}=\sum_{i=1}^{n} \frac{\mathbf{u}_{i}^{T} \mathbf{b} \sigma_{i}}{\sigma_{i}^{2}+\Lambda^{2}} \mathbf{v}_{i}
$$

where $\Lambda$ is the regularization parameter, $\mathbf{u}_{i}$ and $\mathbf{v}_{i}$ are the measurement and the solution modes, and $\sigma_{i}$ are the SVs. This decomposition of the solution of the inverse problem is a direct result of the SVD of the transfer matrix, based on point-source responses. Based on the fact that the mode vectors $\mathbf{u}_{i}$ and $\mathbf{v}_{i}$ are unitary, Eq. (26) makes clear that the constant $\Lambda$ and the values of $\sigma_{i}$, balance the participation of solution modes $\mathbf{v}_{i}$ in the estimate $\mathbf{x}_{\Lambda}$. The solution modes are ordered by the SVD with respect to spatial frequency content, from low to high, as depicted in Figs. 10(a) and 10(b). From a signal theory point of view, a good imager would be susceptible to all spatial frequencies with nearly equal amplitude. Examples are the flat frequency response of professional loudspeakers or the gradually decreasing modulation transfer function of a microscope in a partially coherent imaging mode. In other words, it would be good if the SVs associated with the solution modes show a slow decline and a small ratio between the largest and the smallest participating SV.

The plot in Fig. 11 shows the normalized SVs for the four beam combiners. This SV plot can be separated into three regimes, based on the index $i$, as defined in Section 4:

- incoherent regime: $i=1 \cdots 4$,

- interferometric regime: $i=5 \cdots 110$,

- unsampled regime: $i=111 \cdots 231$.

As stated, a good imager would demonstrate a slow decline in the SVs and a small ratio of the largest (in all cases $\sigma_{1}$ is normalized to 1 ) and the smallest SV. This drop in SV amplitude can be split in the vertical measures $A$ and $B$, as indicated in Fig. 11. We can now analyze the curves as follows. In terms of a flat frequency response, the classical HM and MI beam combiners perform well. The height difference between these two curves, $A_{M I}$ versus $A_{H M}$, relates to the responsiveness of the very loworder modes. Apparently, for the MI imager, the very lowfrequency nature of the source (imaged on the six large pixels) is relatively much stronger represented than the fine structure of the source. As for the HM response, there is a large drop over the first few values. This can be related to the fact that the low-frequency response is present in every measurement at baseline length $B_{\text {in }}^{(k)}$. The average levels of the second regimes are different for the MI and HM lines. This is due to the nature of the measurement signals. A typical MI signal shows an extended incoherent signal (related to low spatial frequencies $)$ of length $\left(d_{\max }-d_{\min }\right) \gg l_{\text {coh }}$ within which a relatively very short fringe packet of length $l_{\text {coh }}$ is present. In the HM case - with field invariant point response functionsthe high-frequent information is present all over the signal, for $\theta \simeq \theta_{\mathrm{in}}^{(\min )} \cdots \theta_{\mathrm{in}}^{(\max )}$.
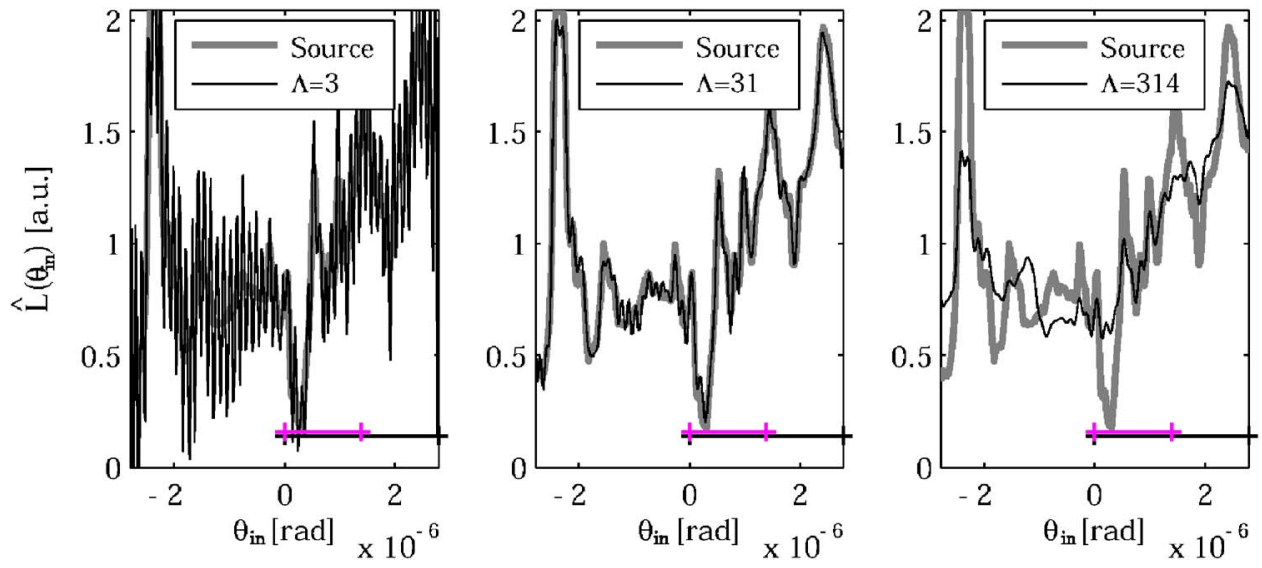

Fig. 9. (Color online) Reconstructions or estimates $\hat{L}\left(\theta_{\text {in }}\right)$ for three regularization parameters $\Lambda=\Lambda_{\text {opt }}\left(\frac{1}{10}, 1,10\right)$, where $\Lambda_{\text {opt }}$ was automatically found using the L curve. In the figure, the size of the detector pixels in angular measure is indicated (short horizontal line), as well as the approximate size of the single-dish-diffraction envelope (long horizontal line). The coaxial MI interferometer that was simulated here, is able to recover spatial details in $L\left(\theta_{\text {in }}\right)$ that are much smaller than the incoherent diffraction limit. A complete set of baselines $B_{\text {in }}^{(k)}$ was taken. 

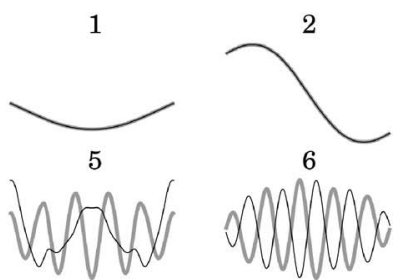

6

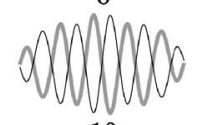

9

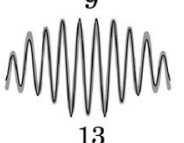

13

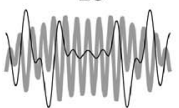

17

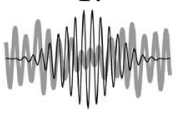

(a)
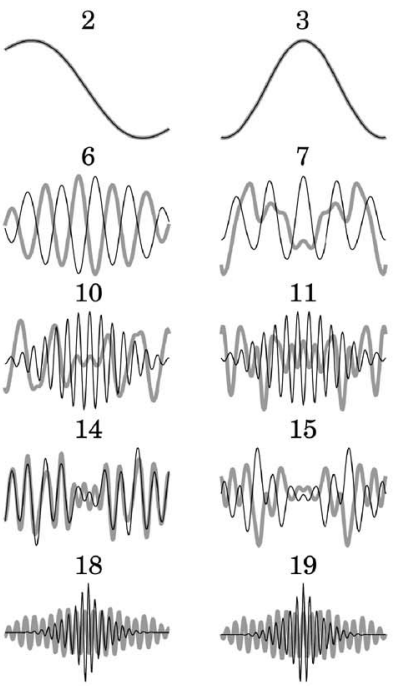

7

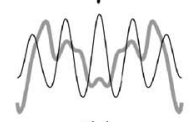

11

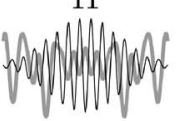

15
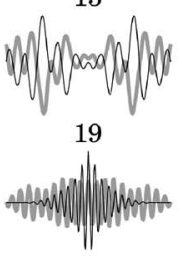

Homothetic
-Densified

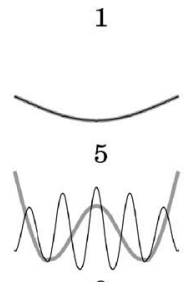

9

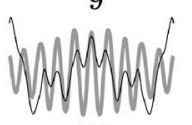

13

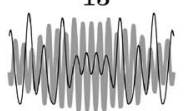

17

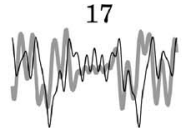

(b)

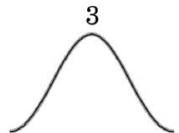

7

6

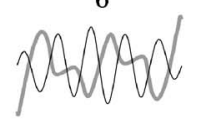

10

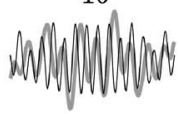

14

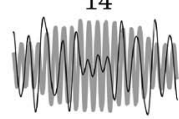

18

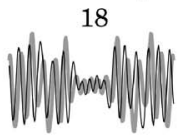

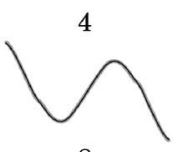

8

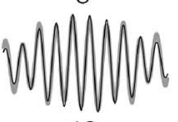

12

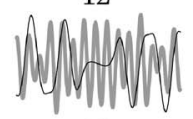

16

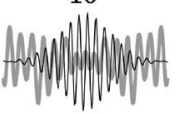

20

4

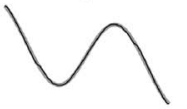

8

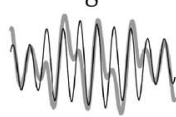

12

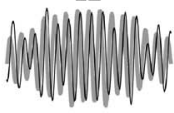

16

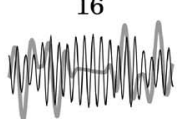

20

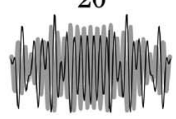

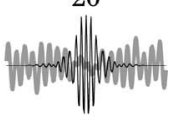

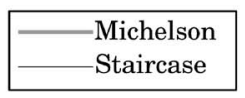

Fig. 10. (a) Comparison of the first 20 solution modes $\mathbf{v}_{i}$ for the HM and the densified methods. Note how the FOV narrows down in the densified case, as the spatial frequency increases. (b) Comparison of the first 20 solution modes $\mathbf{v}_{i}$ for the MI and the staircase methods. Generally, the modes are a sum of several harmonics. Note the occurrence of symmetrical and antisymmetrical modes, e.g., in the staircase modes 5 and 6.

Compared with the relatively flat interferometric frequency response of the HM and MI beam combiners, the beam combiners staircase and densified show steeper declines, defined as $\mathrm{B} /\left(i_{B}-i_{\mathrm{PSFs}}\right)$, for the $\mathrm{SVs}$ in the interferometric regime. The curve for densified is not even reaching the spatial frequencies related to the maximum baseline $B_{\text {in,max }}$. The SV plot actually shows how the densified FOV narrows down for higher spatial frequencies, which is also visible in the reconstruction modes $\mathbf{v}_{i}$ in Fig. 10. Clearly, more steps $d$ are necessary for the densified simulation to present a proper frequency transfer-which was intentionally not done for illustrative purposes. The figure hence shows that the design of the beam combiner

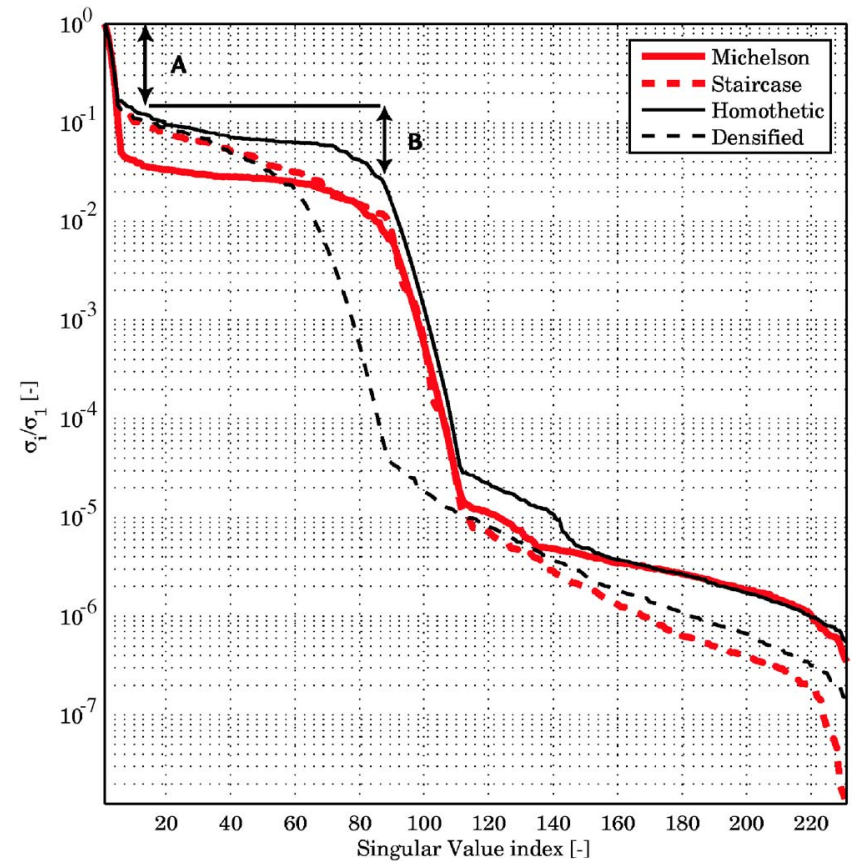

Fig. 11. (Color online) Normalized SVs $\sigma_{i} / \sigma_{1}$ for the four interferometry methods. A flat line up to the cutoff induced by the maximum baseline $B_{\text {in,max }}(\sim i=100)$ indicates a proper transfer of all spatial frequencies. See text for details.

and the intended set of observations of the stellar source can be checked for transferring properly all spatial information of the stellar source.

The staircase transfer is slowly degrading up to the frequency limit related to $B_{\mathrm{in} \text {,max }}$. This means that the higher spatial frequencies are relatively more susceptible to loss of signal strength induced by the staircase mirror. A possible source for this inefficiency is that the folding of the fringe signal destroys the unique relation between source $L\left(\theta_{\text {in }}\right)$ and measurement $I_{j k}(d)$. Apparently, this effect gets worse for increasing baseline lengths.

\section{CONCLUSIONS}

The interferometric imaging process can be expressed as a matrix-vector multiplication $\mathbf{b}=\mathbf{A} \cdot \mathbf{x}$. By doing so, the forward expression gives insight in the construction of partially coherent detection signals since generally speaking, fringed intensity signals with slightly different origins are summed. The inversion of this equation, necessary to perform imaging with measured fringe signals, needs regularization.

With the regularization tools in place, optimal estimates can automatically be obtained from photon-starved or otherwise degraded interferometric measurements. The transfer of stellar information through any of the four presented interferometers into interferometric signals can be described and stored in a single transfer matrix $\mathbf{A}$. For various sources $L\left(\theta_{\text {in }}\right)$ and changing observation times, each interferometer type-related $\mathbf{A}$ can produce simulated measurements, from which optimal reconstructions $\hat{L}\left(\theta_{\text {in }}\right)$ can be produced.

The transfer matrices are constructed under the assumption that the beam relay is perfectly known and no 
perturbations exist in the optics or the detector. Further investigation is required to check the reconstruction performance when the delay $d$ and the baselines $B_{\text {out }}$ and $B_{\text {in }}$ are known with finite accuracy. Insensitivity to inaccuracies in the knowledge of these parameters may outweigh performance advantages of the configurations as they are currently simulated. However, it should be mentioned that noise in the transfer matrix is essentially treated the same as noise in the measurement vector; the presented inversion algorithm will still provide solutions that fit the measurements best.

Despite the different optical layouts of the interferometers, the singular value analysis of each matrix $\mathbf{A}$ shows that the same features appear in the reconstructions and that the interferometers, or chosen system parameters for equal interferometers, can be compared on their imaging merits. Preliminary analyses in the photon-starved regime have shown that a homothetic imager, indeed resembling a true imager the most, performs superior to the other three, with the staircase imager as runner-up. An investigation based solely on the signal-to-noise ratio in detection signals would neglect the existence of large incoherent parts in each detected signal. Extensive simulations of these interferometers, with a selected stellar source as an object and using DARWIN-like telescope diameters and separations, are projected for the near future.

\section{ACKNOWLEDGMENTS}

This work is part of the SRON activities in developing an imaging mode for the DARWIN nulling array and is a result of the Ph.D. research performed by C. van der Avoort.

Corresponding author C. van der Avoort's e-mail address is cas.van.der.avoort@nxp.com.

\section{REFERENCES}

1. A. Quirrenbach, "Optical interferometry," Annu. Rev. Astron. Astrophys. 39, 353-401 (2001).

2. S. K. Saha, "Modern optical astronomy: technology and impact of interferometry," Rev. Mod. Phys. 74, 551-600 (2002).

3. P. M. Hinz, J. R. P. Angel, D. W. McCarthy, Jr., W. F. Hoffman, and C. Y. Peng, "The Large Binocular Telescope Interferometer," Proc. SPIE 4838, 108-112 (2003).

4. J. E. Baldwin and C. A. Haniff, "The application of interferometry to optical astronomical imaging," Philos. Trans. R. Soc. London, Ser. A 360, 969-986 (2002).

5. A. Labeyrie, "Resolved imaging of extra-solar planets with future $10-100 \mathrm{~km}$ optical interferometric arrays," Astron. Astrophys., Suppl. Ser. 118, 517-524 (1996).

6. I. Montilla, S. F. Pereira, and J. J. M. Braat, "Michelson wide-field stellar interferometry: principles and experimental verification," Appl. Opt. 44, 328-336 (2005).

7. DARWIN The Infrared Space Interferometer: Redbook (ESA-SCI, 2000), Vol. 12.

8. A. A. Michelson, "On the application of interference methods to astronomical measurements," London, Edinburgh Dublin Philos. Mag. J. Sci. 30, 1-21 (1890).

9. A. A. Michelson and F. G. Pease, "Measurement of the diameter of Alpha Orionis with the interferometer," Astrophys. J. 53, 249 (1921).

10. M. A. Born and E. Wolf, Principles of Optics, 6th ed. (Pergamon, 1980), pp. 508-510.

11. M. Young and P. D. Hale, "Off-axis illumination and its relation to partial coherence," Am. J. Phys. 63, 1136-1141 (1995).

12. A. N. Tikhonov and V. Y. Goncharsky, Solutions of Ill-Posed Problems (Winston \& Sons, 1977).

13. P. C. Hansen, "Regularization tools: a Matlab package for analysis and solution of discrete ill-posed problems," Numer. Algorithms 6, 1-35 (1994).

14. P. C. Hansen, "The discrete Picard condition for discrete ill-posed problems," BIT 30, 658-672 (1990).

15. P. C. Hansen, "Analysis of discrete ill-posed problems by means of the L-curve," SIAM Rev. 34, 561-580 (1992).

16. R. Visser, "Regularization in nearfield acoustic source identification," in Proceedings of the Eighth International Congress on Sound and Vibration (2001), pp. 1637-1644. 